\title{
MULTIFUNCTIONAL AND LOW COST BRAILLE DISPLAY
}

\author{
Shravya K Holla ${ }^{1}$, Anuradha J.P ${ }^{2}$, Rakshita $\mathbf{M}^{3}$, Varshitha $\mathbf{P}^{4}$, Tejaswini B.J ${ }^{5}$ \\ ${ }^{1}$ Student, department of Electronics \& Communication, B.N.M Institute of Technology \\ ${ }^{2}$ Assistant Professor, department of Electronics \& Communication, B.N.M Institute of Technology \\ ${ }^{3}$ Student, department of Electronics \& Communication, B.N.M Institute of Technology \\ ${ }^{4}$ Student, department of Electronics \& Communication, B.N.M Institute of Technology \\ ${ }^{5}$ Student, department of Electronics \& Communication, B.N.M Institute of Technology \\ Iholla.shravya@gmail.com,${ }^{2}$ anu1572@yahoo.co.in, ${ }^{3}$ rakshitamanojkumar@gmail.com, \\ varshithaprabhakar@gmail.com, ${ }^{5}$ bjtejaswini.u@gmail.com
}

\begin{abstract}
Braille Display is a device which helps the visually impaired to read a text file in Braille format and as well receive an audio output of the text file. It gives an option to the user whether to receive output in terms of braille or audio. Braille is a tactile writing language of raised dots using which blinds and visually impaired people read and write through touch. The pattern of raised dots can be read with the fingers by blinds. The number and arrangement of these dots forms alphabets, numbers and special characters. The braille output in Braille display is observed in Braille text popper device which is made up of a plate having holes. Solenoids are placed under each hole to raise or lower the braille pin which will emerge through perforations on the top plate to form a Braille dot. The perforations serve as guides for the pins and form six dots which is equivalent to one Braille cell. Raspberry Pi Microcontroller is used to convert the text file into Braille format and thus controls the corresponding solenoid present below each of the holes to either pop up or down the hole by sending signal values to solenoids. Thus the solenoids align themselves based on the input character from the text file. The blinds can feel the sense of touch of the Braille pins that are popped up and they recognize the character accordingly. This way, blinds can recognize character by character. At every iteration, three characters from text file comes out in braille in Braille text popper device. The next button would display next three characters and previous button would display previous three characters. If the user prefers to hear audio, then it would give audio output of the text file through ear phones. Thus, the device facilitates the user either to read the text in braille or to hear the text as audio making it multifunctional.
\end{abstract}

Keywords: Braille, Raspberry pi, Solenoids, pyttsx3

\section{INTRODUCTION}

Braille is a language for blinds used for communication and education purposes for blinds. It consists of raised dots arranged in "cells." A cell consists of six dots, arranged in two columns of three dots each that fit under the fingertip. Fig 1 shows a braille cell. Each cell represents a letter, a word, a combination of letters, a numeral or a punctuation mark. The number and arrangement of these dots are different for one character from another. The pattern of raised bumps or dots can be read with the fingers by blinds. Fig 2 shows braille representation of alphabets, punctuation marks and numbers.

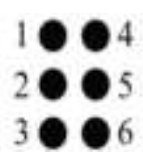

Figure 1. Braille cell

The main objective of the project is to design and develop a Braille output device for the visually impaired individuals that enable them to read the text from computer. However, Braille displays available in the market are highly expensive. The cheapest refreshable braille display 
costs nearly 800 USD, which is far out of reach for most of the people who need devices like this. We have constructed this Braille display in Rs 9060 . According to a recent survey by a national organization for ophthalmologists India accounts for $20 \%$ of the total blind population of the world, with 7.8 million visually impaired out of the 39 million across the globe.

\begin{tabular}{|c|c|c|c|c|c|c|c|c|c|}
\hline \multicolumn{10}{|c|}{ The Bralle Alphabet } \\
\hline$\cdot$ & $=$ & - & $=$ & $\because$ & $=$ & $=$ & $=$ & $\therefore$ & $\therefore$ \\
\hline a & $b$ & c & d & e & $f$ & 9 & $\mathrm{~h}$ & i & I \\
\hline : & $\vdots$ & 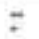 & I & 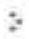 & $\Sigma$ & $\equiv$ & $\$$ & $\dot{q}$ & 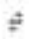 \\
\hline $\mathrm{k}$ & I & $m$ & $\mathbf{n}$ & 0 & p & $q$ & $t$ & $s$ & $t$ \\
\hline \pm & \pm & 4 & $\Xi$ & 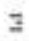 & $\approx$ & & & & \\
\hline$u$ & $\mathrm{v}$ & $w$ & $x$ & $y$ & $z$ & & & & \\
\hline
\end{tabular}

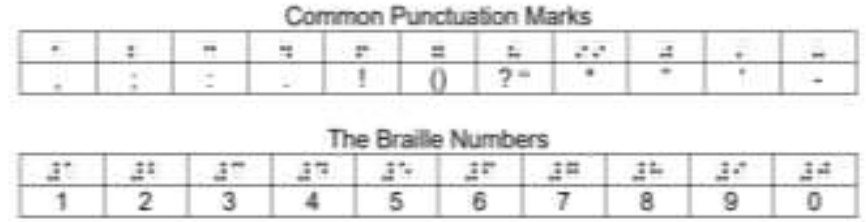

Figure 2. Braille representation [6]

India is now home to the world's largest number of blind people. 285 million people are estimated to be visually impaired worldwide: 39 million are blind and 246 have low vision. About $65 \%$ of all people who are visually impaired are aged 50 and older, while this age group comprises about $20 \%$ of the world's population [7]. Thus, creating a low cost, intuitive text to braille converter is the main purpose of this paper.

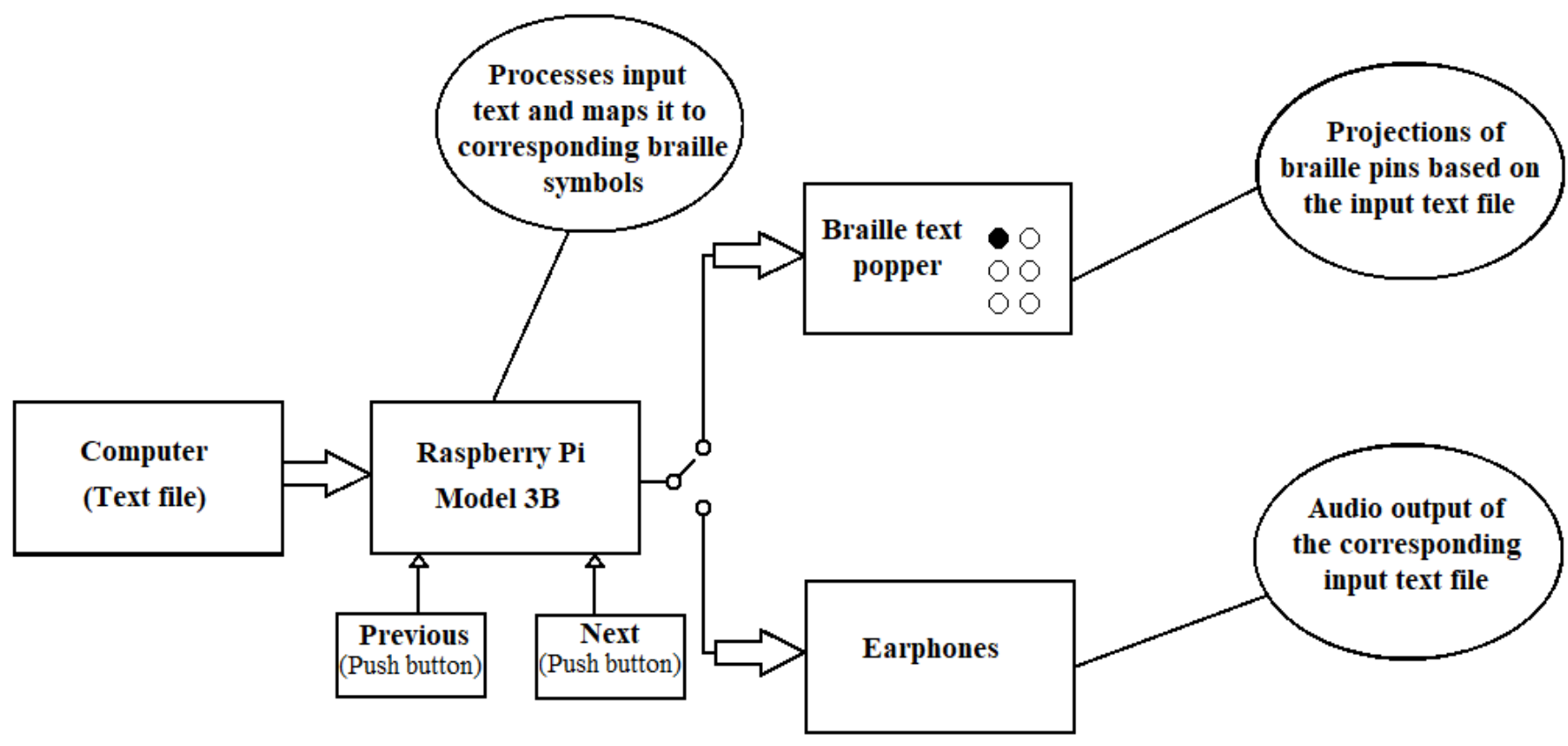

Figure 3. Block diagram

\section{LITERATURE SURVEY}

Among the recent research works, Saurabh Bisht, Sandeep Reddy Goluguri, Rajat Maheshwari, Akhilesh Kumar and P Sathya have proposed a refreshable electronic braille display using Raspberry Pi 2 and Arduino Mega2560 board which converts normal text file as well as printed image files to braille. It makes use of tesseract-ocr engine of Google for converting image files to text. Image processing is done by Raspberry pi and display is driven by Arduino. The display is made of 6 servo motors, which can be controlled by the PWM. The servo motor acts as an actuator in this project [1]. Swati Malik, Preet Jain has proposed a lowcost portable device called "E Braille reader for visually challenged person". A blind person will be able to read E-Books by actually connecting his hardware to certain E-Books. The matter 
from the E-Book is downloaded to his hardware. The matter is then spoken out by the hardware and also, the hardware rotates a disc that has the Braille characters embossed on a disc. The blind person can then feel the Braille from this disc surface [2]. Shahruk Hossain, Abdullah Abyad Raied, Asifur Rahman,Ahsam Rabby Khan,Arnab Bhattacharjee, Celia Shahnaz and Shaikh Anowarul Fattah have proposed a display that generates all Braille characters by simple sliding arrangement of two eleven-slot pre protruded sliders utilizing permutations of the slots. Piezo-electric based displays is used as a actuator that consumes a large amount of power to generate each character by dynamically actuating various slots. The text scanner is presented in the form of a mobile app, built using Google's open source optical character recognition (OCR) engine. [3]. Vinaya Phutak, Richa Kamble, Sharmila Gore, Minal Alve, R.R.Kulkarni proposed a system which does Text To Speech conversion using raspberry-pi. Here, Text to Speech conversion is done using TTS Unit.[4]. Sariat Sulthana, Aaphsaarah Rahman, Fyaz Hasan Chowdhary, Hasan U. Zaman proposed a novel design of a low-cost, low-power, portable and user-friendly Braille system. The designed system takes the input through Braille keyboard and produces the Braille output in Braille display, the corresponding English characters are also displayed on the LCD and also in the laptop if it is connected. It also has the capability of reading documents as text [5].

\section{METHODOLOGY}

\subsection{Description of block diagram}

The device works on two modes namely mode A and mode B. These modes are selected by a toggle switch. Fig3 shows the block diagram. When mode A is selected, the text file from the computer is converted into Braille format. When mode B is selected, an audio output of the text present in the text file is been obtained. Two push buttons is been used. The "Next" push button is to obtain the next three characters in braille text popper. The "Previous" push button is to obtain the previous three characters in braille text popper in braille format.

In mode A the board functions as:

- To produce text in Braille, Raspberry pi receives the text file stored in the computer.

- Raspberry pi then converts each character into Braille symbol and then sends signals to solenoid to actuate the Braille pins.

- The blinds can feel the sense of touch of the Braille pins that are popped up according to the input letter and they recognize the letter accordingly.

In this manner, at every iteration, three characters is displayed in braille text popper device. The whole control mechanism is been programmed in Python language in Raspbian Operating System. In mode B, raspberry pi model 3B gives an audio output of the text file using pyttsx 3 package in Raspbian operating system.

\subsection{Functionality of each component}

- Raspberry pi- Raspberry pi is used to convert a character from the text file in the form of Braille. It sends low signal to the respective solenoid under each pin to be raised. For example, for a character ' $a$ ' according to Braille only the first dot of the cell should be raised. The other five should pop down. So current should be sent to the solenoid below the keys 2, 3, 4, 5 and 6 so that it is pulled down and only the first dot is raised up. The visually impaired people feel and interpret this. In this manner any character can be represented in a Braille display. Thus Raspberry pi maps each character to six different signal levels and sends it to each of the solenoids.

- Braille text popper-Braille text popper is a key setup which has a solenoid below each key. Each solenoid is responsible for lowering and raising a pin, which will emerge through perforations on the top plate to form a Braille dot. The perforations serve as guides for the pins and form six dots which is equivalent to one Braille cell.

- Earphones - Ear phones are used to hear the audio output of the text file. 
- Push buttons - Two push buttons are been used. The "Next" push button is to obtain the next three characters in braille text popper. The "Previous" push button is to obtain the previous three characters in braille text popper in braille format.

- Toggle Switch - Toggle switch is used to select mode A or mode B. It is used to select either the braille format output or audio output.

\subsection{Hardware Description}

1) Raspberry pi model 3B - The Raspberry pi Model 3B is a 64 bit micro-controller. It has 4 USB ports, 1 HDMI port, 1 ethernet port, 1 port for power supply, a micro SD Card slot, an Audio jack and several pins for connecting various other components. Raspberry pi is used to convert a character from the text file in the form of Braille. Fig 4 shows Raspberry pi model 3B.

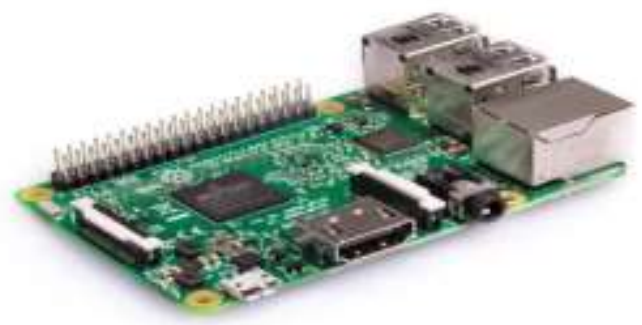

Figure 4. Raspberry pi model 3B [8]

2) Braille text popper - Braille text popper is a key setup which has solenoid below each key. Each solenoid is responsible for lowering and raising a pin, which will emerge through perforations on the top plate to form a Braille dot. The perforations serve as guides for the pins and form six dots which is equivalent to one Braille cell as shown in Fig. 5.

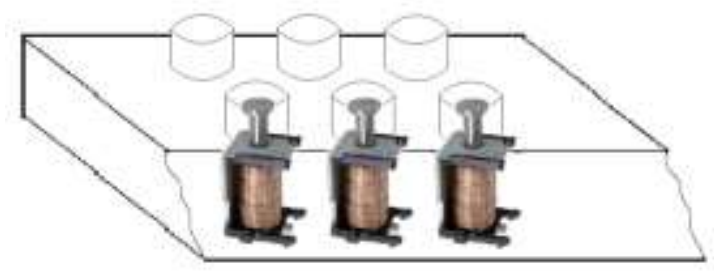

Figure 5. Braille text popper

3) Solenoid- A solenoid is an electromagnet. When an electrical current is passed through the coils windings, it behaves like an electromagnet and the plunger is attracted towards the coil by the magnetic flux setup and pops down. When the supply current is turned "OFF" the electromagnetic field generated previously by the coil collapses and the energy stored in the compressed spring forces the plunger back out to its original rest position. Hence back and forth movement is obtained on sending current signals. Thus electrical energy is converted into linear up and down motion. Thus solenoids align themselves based on the input character from the text file. Fig 6 shows a solenoid. 12 volts solenoid is been used in our device.

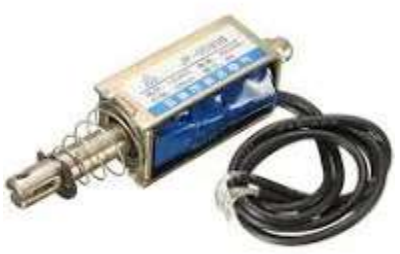

Figure 6. Solenoid [9] 
4) Ear phones - Earphones are used to hear the text in the audio format. Raspberry pi has $3.5 \mathrm{~mm}$ audio jack to which earphones is inserted as shown in Fig 7.

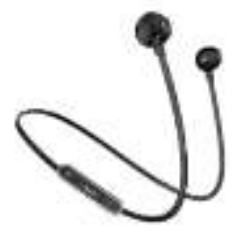

Figure 7. Ear phones [10]

5) Push buttons - A push-button is a simple switch mechanism as shown in Fig 8. In our device, two push buttons is been used. The "Next" push button is to obtain the next three characters in braille text popper. The "Previous" push button is to obtain the previous three characters in braille text popper.

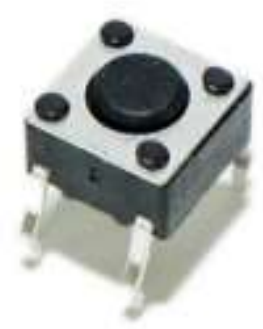

Figure 8. Push button [11]

6) Toggle switch - The toggle switch is a type of electric switch which has two positions ON or OFF as shown in Fig 9. In our device, Toggle switch is used to select mode A or mode B i.e to select either the braille format output or audio output.

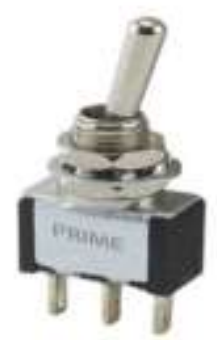

Figure 9: Toggle switch [12]

\subsection{Software Description}

- Raspbian Operating System - Raspbian is a Linux based operating system, which has been specially designed for the Raspberry Pi.

- Python- Python is object-oriented, imperative and functional programming language and is supported by Raspbian Operating System.

- Pyttsx3 text to speech package - Pyttsx 3 is a text-to-speech conversion library in Python which is been used in our project .This package works in Windows, Mac, and Linux and is compatible with python 2 and 3.

\section{EXPERIMENT AND RESULT}

The prototype is developed and desired output is been obtained. The software as well as the hardware is implemented successfully. The text file stored in computer is been converted into Braille format and the audio output is also been obtained. The Braille output is observed in 
Braille popper device. The Braille popper device has solenoids placed under each hole which is responsible for raising or lowering the braille pin. Each braille pin will emerge through perforations on the top plate to form a Braille dot. The perforations serve as guides for the pins and form six dots which is equivalent to one Braille cell. Raspberry Pi Microcontroller converts the text file stored in the computer into Braille format and it also controls the corresponding coil present below each of the holes to pop up or down the hole based on the character in the text file. The blinds can feel the sense of touch of the Braille pins that are popped up and they recognize the letter accordingly. This way, they recognize character by character. At every iteration, three characters from text file comes out in braille format. The next button would display next three characters and previous button would give previous three characters. The toggle switch gives an option of either choosing braille output or audio output. This braille output is been observed in Braille popper device. The audio output is been heard through earphones. Thus the presented braille display is multifunctional as it gives braille and audio output. Fig 10 shows the working setup.

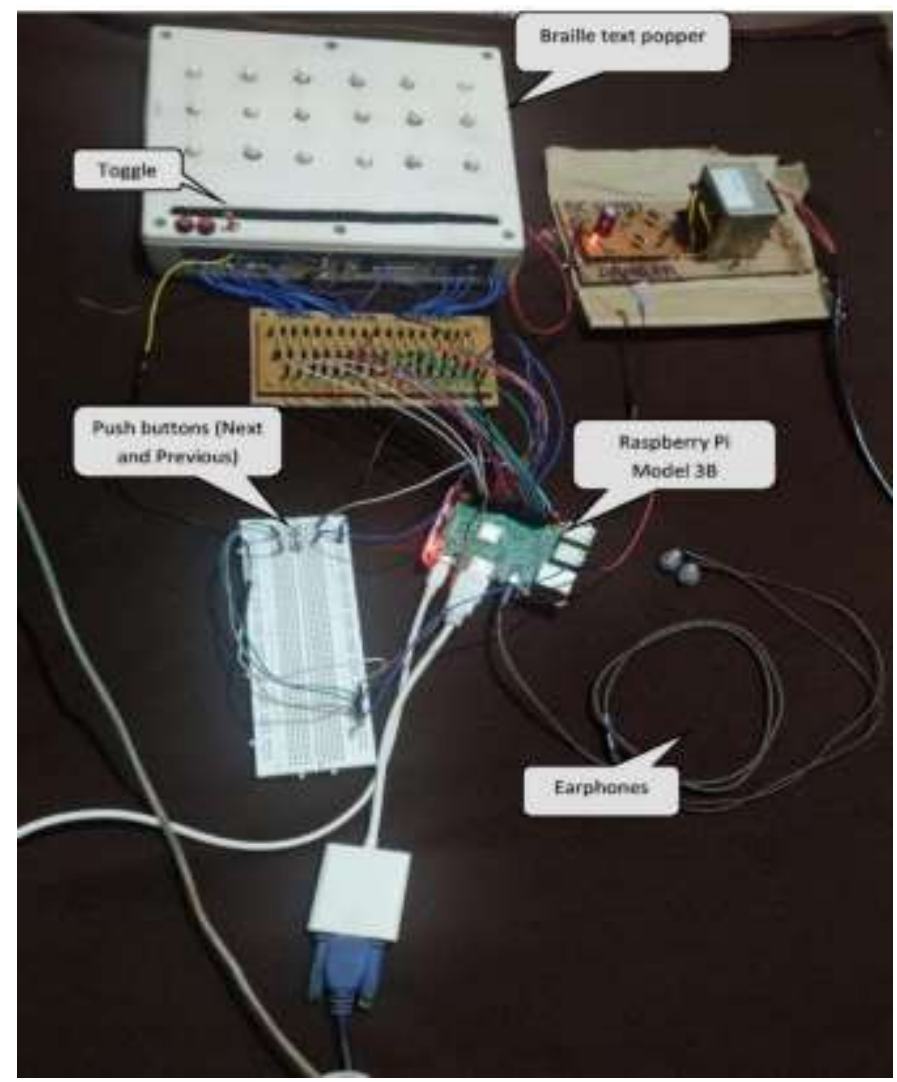

Figure 10. The working setup

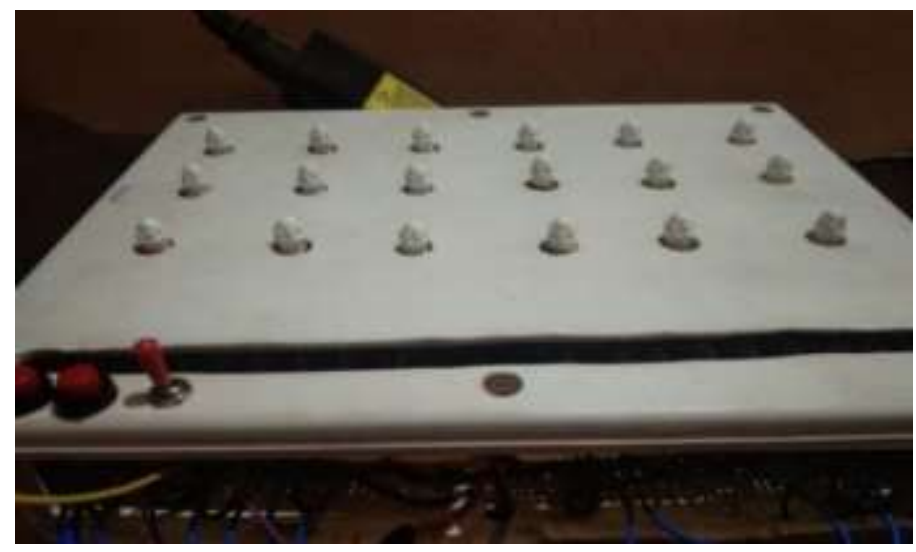

Figure 11. Braille text popper before execution 


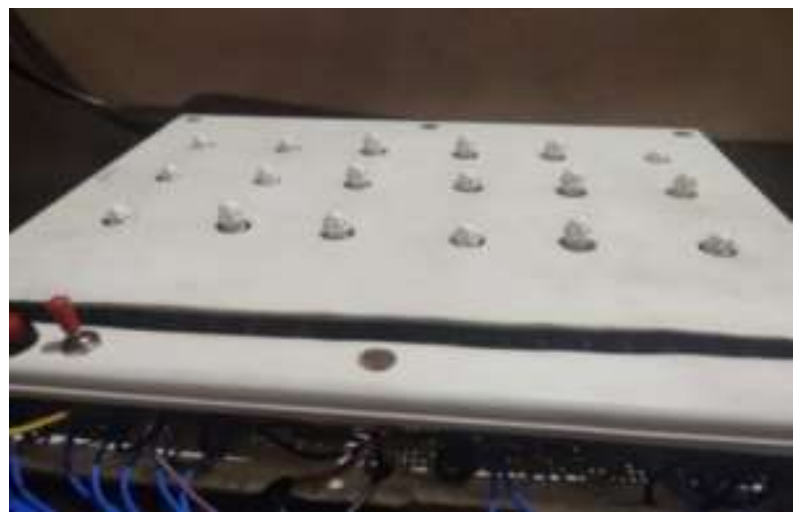

Figure 12. Braille text popper after execution

Fig 11 shows the Braille text popper before execution. Fig 12 shows braille text popper after execution. The solenoids are been aligned based on corresponding input text. In this figure, "Pr" is been represented in Braille format whose standard braille representation is as shown in Fig 13. Fig 14 shows internal view of Braille text popper.

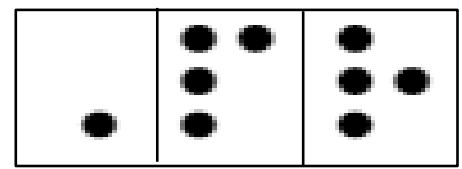

Figure 13. Braille representation of ' $\mathrm{Pr}$ '

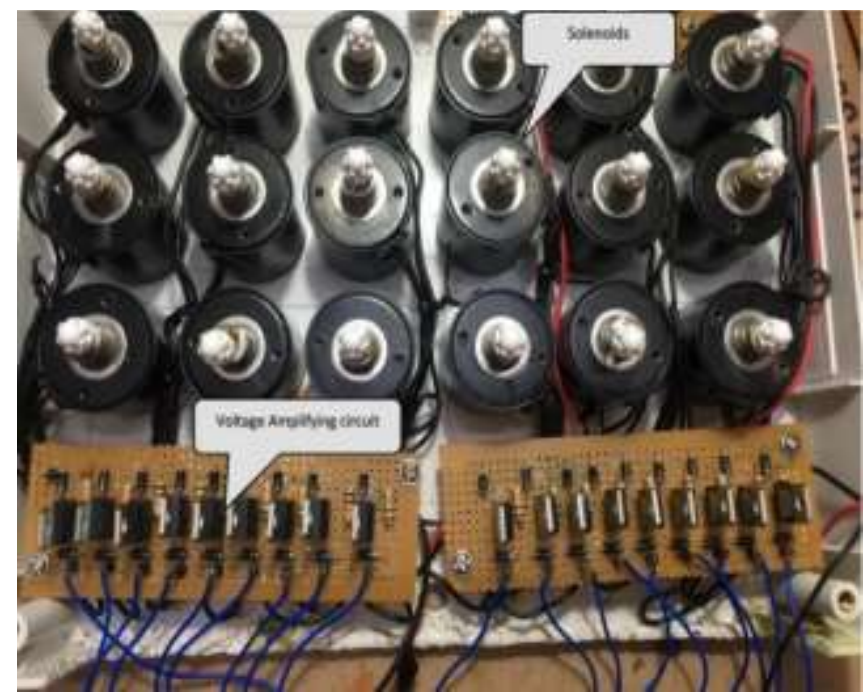

Figure 14. internal view of braille text popper

Number representation in Braille text popper number- 12

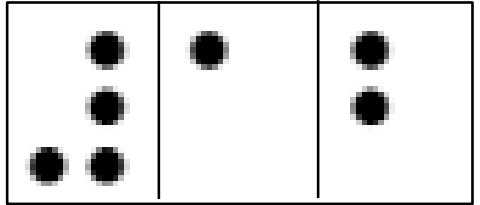

Figure 15. Braille representation of "12" 


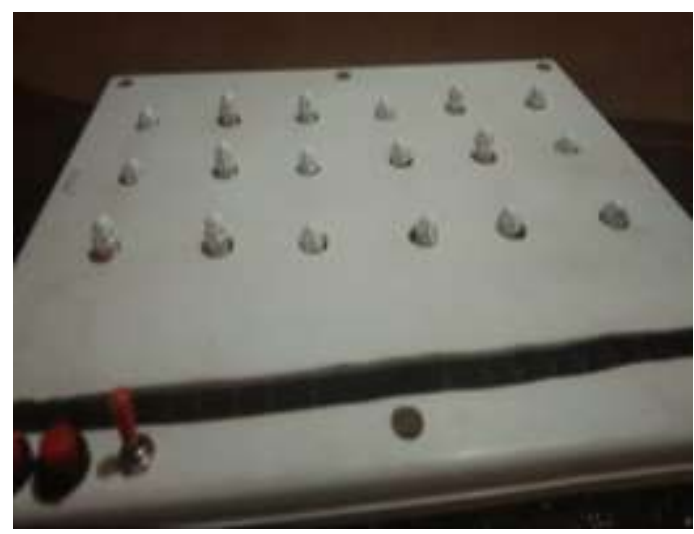

Figure 16. "12" represented in Braille text popper

Fig 15 shows Braille representation of the "12". Fig 16 shows the Braille text popper showing " 12 ".

\section{Punctuation marks representation in braille text popper}

sequence - .,!?:;

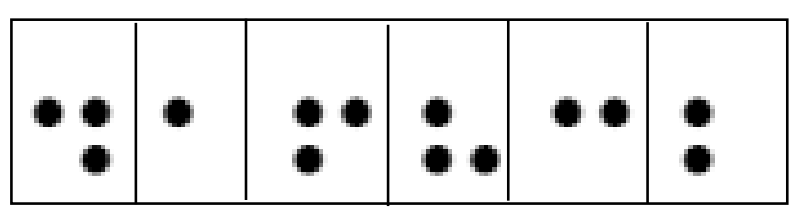

Figure 17. Braille representation of the characters “.,!?:;”

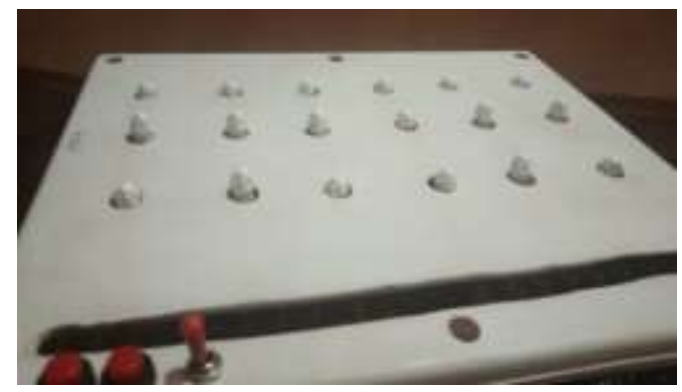

Figure 18. Characters “.,!" represented in Braille text popper

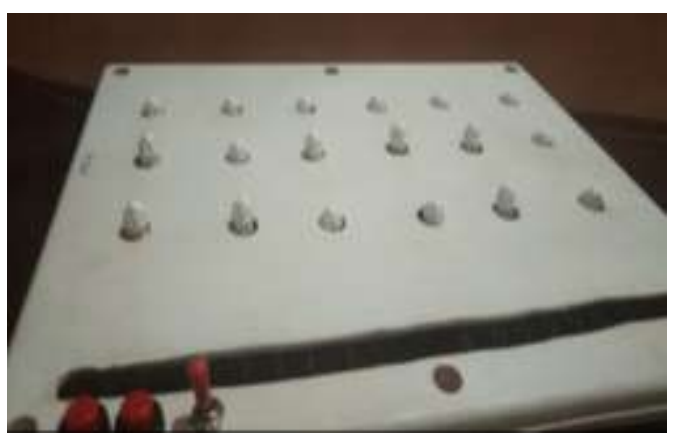

Figure 19. characters "?:;" represented in Braille text popper

Fig 17 shows Braille representation of the characters ".,!?:;”. Fig 18 shows the Braille text popper showing “.,!" and Fig 19 shows the Braille text popper showing "?:;"”

\section{CONCLUSION}

This project proposes a user-friendly Braille display. We have developed a Braille display which acts as text to braille converter as well as text to audio converter. The hardware and software implementation is completed successfully. Text file stored in the computer is converted into braille format and as well to audio form. At every iteration, three characters from text file 
comes out in braille in Braille text popper device. The next button would display next three characters and previous button would display previous three characters from the file. If the user prefers to hear audio, then it would give audio output of the text file through ear phones. Thus the device can be called as multifunctional as it facilitates the user either to read the text in braille as well as to hear the text in audio form. We have constructed this Braille display in Rs 9060. Braille displays available in the market are highly expensive. The braille display available in the market is nearly $800 \mathrm{USD}$, which is far out of reach for most of the people who need devices like this. Thus this device is not only multifunctional but also cost effective and best suits the title "Multifunctional and low cost Braille display". This device will help the visually impaired to be independent and flourish in this fast- developing world.

\section{FUTURE ENHANCEMENT}

In future works, E-Book reader for blinds can be developed. An application must be developed which takes the voice input of the name of the book specified by the user which the user wants to read. The application is supposed to download the respective book from the internet and the book should then be converted into Braille format. The user can feel the Braille by using Braille text popper. If the user wants audio output then the blind person can hear it through earphones.

\section{REFERENCES}

[1] Saurabh Bisht, Sandeep Reddy Goluguri, Rajat Maheshwari, Akhilesh Kumar and P Sathya.:"Refreshable Braille Display Using Raspberry Pi and Arduino", International Journal of Current Engineering and Technology, Vol.6, No.3, E-ISSN 2277-4106, P-ISSN 2347-5161, June 2016.

[2] Swati Malik, Preet Jain: "Low Cost Portable E-Braille for Blind and visually impaired Persons", International Journal of Innovative Research in Computer and Communication Engineering, Vol.4, Issue 12, December 2016.

[3] Shahruk Hossain, Abdullah Abyad Raied, Asifur Rahman,Zaowad Rahabin Abdullah,Dipanjan Adhikary,Ahsam Rabby Khan,Arnab Bhattacharjee,Celia Shahnaz, Shaikh Anowarul Fattah,'Text to Braille Scanner with Ultra Low Cost Refreshable Braille Display" IEEE, ISSN-978-1-5386-5566-5/18, 2018.

[4] P.V.N Reddy :’Text to Speech Conversion Using Raspberry-Pi for Embedded System”, International Journal of Innovative Research in Science, Engineering and Technology, Vol.1, Issue 1, November 2012.

[5] Sariat Sulthana, Aaphsaarah Rahman, Fyaz Hasan Chowdhary, Hasan U Zaman, International Conference on Intelligent Computing, Instrumentation and Control Technologies, IEEE, ISSN- 978-1-5090-6106-8/17, 2017.

[6]https://images.app.goo.g1/JgkuSnNqzoyij2oRA

[7] https://www.who.int/blindness/publications/globaldata/en/

[8]https://images.app.goo.gl/JigSfPYo2HtWHYbN7

[9] https://banggood.app.link/GB3vKsTVW1 (solenoid)

[10] https://images.app.goo.gl/Tmvrcaqjxc6Ybbvu6

[11]https://images.app.goo.gl/MzPwXmezuCY84VL17

[12] https://images.app.goo.gl/wgo6iVVKedzvFgmz7 